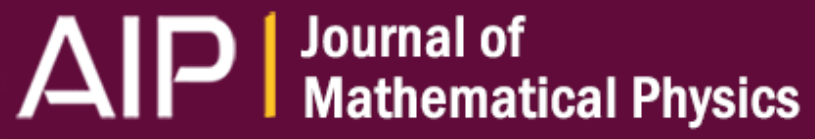

\section{Reducing the number of questions in nonlocal games}

M. Junge, T. Oikhberg, and C. Palazuelos

Citation: Journal of Mathematical Physics 57, 102203 (2016); doi: 10.1063/1.4965831

View online: http://dx.doi.org/10.1063/1.4965831

View Table of Contents: http://scitation.aip.org/content/aip/journal/jmp/57/10?ver=pdfcov

Published by the AIP Publishing

\section{Articles you may be interested in}

Game-theoretic characterization of antidegradable channels

J. Math. Phys. 55, 092202 (2014); 10.1063/1.4895918

Strategies and payoffs in quantum minority games

AIP Conf. Proc. 1327, 477 (2011); 10.1063/1.3567477

Understanding quantum non-locality through pseudo-telepathy game

AIP Conf. Proc. 864, 228 (2006); 10.1063/1.2400894

Distributivity breaking and macroscopic quantum games

AIP Conf. Proc. 750, 108 (2005); 10.1063/1.1874564

Dynamics of reduced density matrices: Classical memory versus quantum nonlocality

J. Chem. Phys. 109, 2994 (1998); 10.1063/1.476891

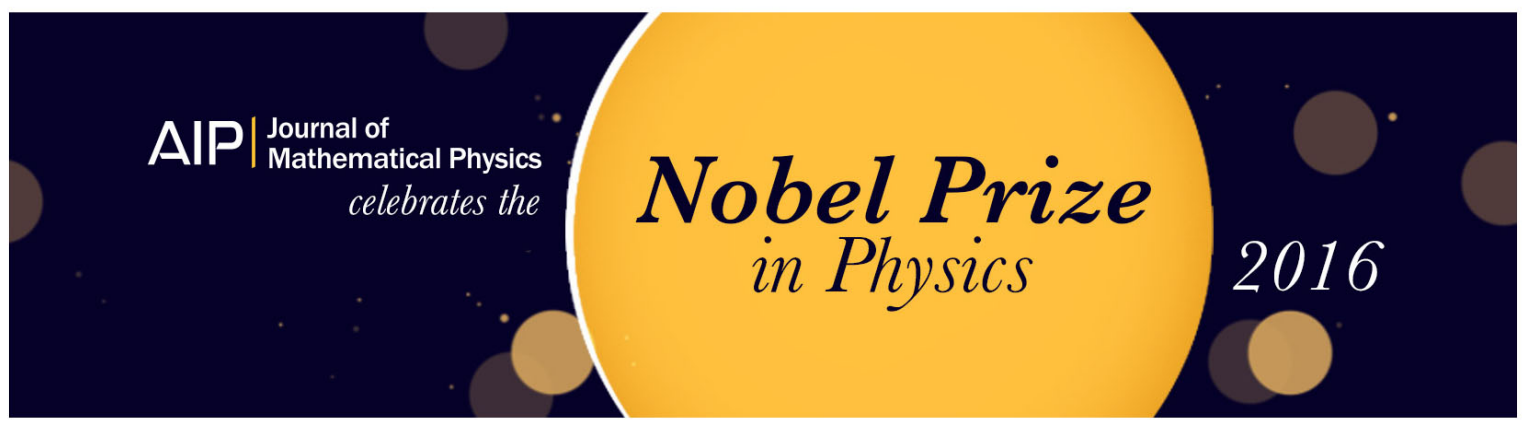




\title{
Reducing the number of questions in nonlocal games
}

\author{
M. Junge, ${ }^{1, a)}$ T. Oikhberg, ${ }^{1, b)}$ and C. Palazuelos ${ }^{2, c)}$ \\ ${ }^{1}$ Department of Mathematics, University of Illinois at Urbana-Champaign, \\ 1409 W. Green St., Urbana, Illinois 61891, USA \\ ${ }^{2}$ Instituto de Ciencias Matemáticas, ICMAT, Facultad de Ciencias Matemáticas, \\ Universidad Complutense de Madrid, Plaza de Ciencias s/n, 28040 Madrid, Spain
}

(Received 24 August 2016; accepted 9 October 2016; published online 28 October 2016)

\begin{abstract}
We show how a vector-valued version of Schechtman's empirical method can be used to reduce the number of questions in a nonlocal game $G$ while preserving the quotient $\beta^{*}(G) / \beta(G)$ of the quantum over the classical bias. We apply our method to the Khot-Vishnoi game, with exponentially many questions per player, to produce a family of games indexed in $n$ with polynomially many $\left(N \approx n^{8}\right)$ questions and $n$ answers per player so that the ratio of the quantum over the classical bias is $\Omega\left(n / \log ^{2} n\right)$. Published by AIP Publishing. [http://dx.doi.org/10.1063/1.4965831]
\end{abstract}

\section{INTRODUCTION AND MAIN RESULT}

A remarkable feature of quantum mechanics is the fact that two observers, each holding half of an entangled quantum state, can perform suitable measurements to produce some probability distributions which cannot be explained by a local hidden variable model. This was first showed by Bell, ${ }^{2}$ based on a previous intuition of Einstein, Podolsky, and Rosen in Ref. 8, and the experimental verification of this phenomenon ${ }^{1,9}$ provides the strongest evidence that nature does not obey the laws of classical mechanics.

A Bell experiment can be understood by means of the so-called nonlocal games, which makes this setting very interesting for computer scientists too. In a bipartite game $G$ (formally, two-prover one-round game), Alice and Bob are asked questions $x$ and $y$, respectively, according to a fixed and known probability distribution $\pi$, and they are required to answer outputs $a$ and $b$, respectively. Let us assume that $x, y \in\{1, \ldots, N\}$ and $a, b \in\{1, \ldots, K\}$, although the setting could be more general. For each pair of questions $(x, y) \in[N] \times[N]$ and pair of answers $(a, b) \in[K] \times[K]$, there is a known probability $V(x, y, a, b) \in[0,1]$ of winning the game, so that the game $G$ is completely determined by $\pi$ and $V$. Then, the aim of the players is to maximize the bias of the game, ${ }^{24}$ defined as the absolute value of the difference between the probability of winning $P_{\text {win }}$ and the probability of losing $P_{\text {los }}$ : bias $=\left|P_{\text {win }}-P_{\text {los }}\right|=\left|2 P_{\text {win }}-1\right|$. Note that this quantity equals $2\left|P_{\text {win }}-\frac{1}{2}\right|$, so the bias measures the deviation of the winning probability from $1 / 2$. To maximize the bias, Alice and Bob can agree on a strategy before the game starts, which is completely described by the numbers $P(a, b \mid x, y)$ giving the probability of answering the couple $(a, b)$ if they are asked the couple $(x, y)$, but they are not allowed to communicate to each other once the game has started.

A classical strategy $P$ for Alice and Bob is given by some functions $f_{A}:[N] \rightarrow[K], f_{B}$ : $[N] \rightarrow[K]$ so that we define $P(a, b \mid x, y)=1$ if $f_{A}(x)=a$ and $f_{B}(y)=b$ and $P(a, b \mid x, y)=0$ otherwise. Then, the classical bias of the game $\beta(G)$ is defined as the largest bias of the game when the players use classical strategies. A quantum strategy between Alice and Bob is of the form $P(x, y \mid a, b)=\operatorname{tr}\left(E_{x}^{a} \otimes F_{b}^{y} \rho\right)$ for every $x, y, a, b$, where $\left\{E_{x}^{a}\right\}_{x, a}$ is a set of non-negative operators acting on a Hilbert space $\mathcal{H}_{A}$ such that $\sum_{a} E_{x}^{a}=\mathbb{1}$ for every $x$ (and analogously for $\left\{E_{y}^{b}\right\}_{y, b}$ ) and $\rho$

\footnotetext{
a) junge@math.uiuc.edu

b) oikhberg@illinois.edu

c) carlospalazuelos@mat.ucm.es
} 
is a density operator (non-negative operator with trace one) acting on $\mathcal{H}_{A} \otimes \mathcal{H}_{B}$. The quantum bias of the game $\beta^{*}(G)$ is defined as the largest bias when the players use quantum strategies.

The existence of quantum probability distributions which cannot be explained by a local hidden variable model is equivalent to the existence of certain games for which the quantum bias is strictly larger than the classical bias. A famous example is given by the CHSH game, ${ }^{6}$ based on the CHSH inequality, ${ }^{5}$ where each player is asked a random bit $(N=2)$ and they must reply with one bit each $(K=2)$. The players win the game if and only if the XOR of the answers is equal to the AND of the questions. Note that here $V(x, y, a, b) \in\{0,1\}$ for every $x, y, a, b$. It is well known that the classical bias is at most $1 / 2$, while the quantum bias is $1 / \sqrt{2} \approx 0.707$.

The aim of this work is to study how much quantum mechanics can deviate from classical mechanics, and a natural way to quantify this deviation is via the quotient $\beta^{*}(G) / \beta(G)$. This quantity has been deeply studied during the last years and, beyond its theoretical interest, it has been shown to be very useful regarding its applications to different contexts such as dimension witnesses, communication complexity, the study of quantum nonlocality in the presence of noise or/and detector inefficiencies, and so on (see Ref. 12 and the references therein). In fact, in order to consider all relevant parameters in the problem, we will denote by $\beta_{d}^{*}(G)$ the quantum bias of the game $G$ when the players are restricted to the use of $d$-dimensional quantum states $\rho$ in the corresponding quantum strategies, so that $\beta^{*}(G)=\sup _{d} \beta_{d}^{*}(G)$. It was proved in Ref. 10 (see also Refs. 14 and 17) that there is a universal constant $C>0$ such that for every bipartite game $G$ with $N$ questions and $K$ answers per player we have

$$
\frac{\beta_{d}^{*}(G)}{\beta(G)} \leq C \ell, \quad \text { where } \quad \ell=\min \{N, K, d\} .
$$

A prominent example of a (family of) game $\left(G_{n}\right)_{n}$ leading to a large quotient $\beta^{*}\left(G_{n}\right) / \beta\left(G_{n}\right)$ was given in Ref. 4, where the authors showed that the so-called Khot-Vishnoi game $G_{K V}^{n},{ }^{13}$ defined with $N=2^{n} / n$ questions and $K=n$ answers per player ( $n$ is a power of 2), leads to a quotient $\beta_{n}^{*}\left(G_{K V}^{n}\right) / \beta\left(G_{K V}^{n}\right) \geq C n / \log ^{2} n$, where $C$ is a universal constant. ${ }^{25}$ According to (1.1), this example is essentially optimal in the number of answers $K$ and in the dimension of the Hilbert space $d$. However, the number of questions is exponentially far away from the best known upper bound $O\left(n / \log ^{2} n\right)$. Our main theorem states that the same estimate can be obtained with polynomially many questions.

Theorem 1.1. There exists a family of games $\left(G_{n}\right)_{n=2^{k}, k \in \mathbb{N}}$ with $N \approx n^{8}$ questions and $n$ answers per player such that

$$
\frac{\beta_{n}^{*}\left(G_{n}\right)}{\beta\left(G_{n}\right)} \geq C \frac{n}{\log ^{2} n}
$$

for a certain universal constant $C$.

To state Theorem 1.1 in a different way, set $\gamma=\beta^{*}\left(G_{n}\right) / \beta\left(G_{n}\right)$. The theorem shows that the "violation" $\gamma$ can be obtained by using an almost optimal number of answers, and almost optimal dimension of the Hilbert space involved $\left(n=O\left(\gamma \log ^{2} \gamma\right)\right)$, as well as polynomially many questions $\left(N=O\left(n^{8}\right) \sim O\left(\gamma^{8} \log ^{16} \gamma\right)\right)$. This is an improvement over Ref. 4 where the number of questions was exponential in $\gamma$ (we are also partially answering some of the open problems posed in Ref. 4 [Section 1.3]).

Decreasing the number of parameters yielding a certain outcome (in our case, the number of inputs allowing to witness a large violation of Bell inequalities) is in general a difficult problem. Passing from exponentially to polynomially many parameters is usually understood as an improvement in the scale of the problem.

We must note that in Ref. 10 the authors gave an example of a family of nonlocal games $G_{J P}^{n}$ with $n$ questions and $n$ answers per player for which $\beta_{n}^{*}\left(G_{J P}^{n}\right) / \beta\left(G_{J P}^{n}\right) \geq \sqrt{n} / \log n$. This is only quadratically far from the best upper bounds in all the parameters of interest at the same time. Hence, the key point in Theorem 1.1 is that it preserves the optimality of the family $\left(G_{K V}^{n}\right)_{n}$ shown in Ref. 4. In fact, the $n$-dimensional quantum state used in Ref. 4, as well as in Theorem 1.1 , to obtain the corresponding lower bound for $\beta_{n}^{*}(G)$ is the maximally entangled state; and it is 
known ${ }^{16}$ that in this case the corresponding quotient $\beta_{n}^{*}(G) / \beta(G)$ is upper bounded by $C \frac{n}{\sqrt{\log n}}$, for a universal constant $C$. So the logarithmic factor cannot be removed.

The main reason to work with the bias of a game $G$ rather than with its value is that in the former case we can adopt a much more general point of view. More precisely, for a family of real coefficients $\left(M_{x, y}^{a, b}\right)_{x, y ; a, b}$, we define the Bell functional $M$ acting on the set of strategies by means of the dual action $\langle M, P\rangle:=\sum_{x, y ; a, b} M_{x, y}^{a, b} P(a, b \mid x, y)$. Then, we define its classical and quantum value, respectively, as

$$
\omega(M)=\sup _{P \in \mathcal{P}_{c}}|\langle M, P\rangle| \quad \text { and } \quad \omega^{*}(M)=\sup _{P \in \mathcal{P}_{q}}|\langle M, P\rangle|,
$$

where $\mathcal{P}_{c}$ and $\mathcal{P}_{q}$ denote, respectively, the set of classical and quantum strategies defined above. It is not difficult to see that there is a one-to-one correspondence between the quotient $\beta_{n}^{*}(G) / \beta(G)$ between the quantum and the classical bias of games and the quotient $\omega_{n}^{*}(M) / \omega(M)$ between the quantum and the classical value of Bell functionals. We will explain this point in more detail in Section II.

The relevance of Equation (1.2) is that it allows to connect the study of Bell inequalities and nonlocal games to the theory of operator spaces. ${ }^{17}$ In particular, if we realize $M=\sum_{x, y, a, b} M_{x, y}^{a, b}\left(e_{x}\right.$ $\left.\otimes e_{a}\right) \otimes\left(e_{y} \otimes e_{b}\right)$ as an element in $\ell_{1}^{N}\left(\ell_{\infty}^{K}\right) \otimes \ell_{1}^{N}\left(\ell_{\infty}^{K}\right)$, one can see that

$$
\omega(M) \approx\|M\|_{\ell_{1}^{N}\left(\ell_{\infty}^{K}\right) \otimes_{\epsilon} \ell_{1}^{N}\left(\ell_{\infty}^{K}\right)} \text { and } \quad \omega^{*}(M) \approx\|M\|_{\ell_{1}^{N}\left(\ell_{\infty}^{K}\right) \otimes_{\min } \ell_{1}^{N}\left(\ell_{\infty}^{K}\right)},
$$

where here $\epsilon$ denotes the injective tensor norm when $\ell_{1}^{N}\left(\ell_{\infty}^{K}\right)$ is seen as a complex Banach space and $\min$ denotes the minimal tensor norm for which one must understand $\ell_{1}^{N}\left(\ell_{\infty}^{K}\right)$ as an operator space. ${ }^{20}$ The symbol $\approx$ can be shown to be an equality if the element $M$ has positive coefficients (in particular, when $M$ is a game). Otherwise, the relation is more subtle. However, the important point for us is that finding an element $M$ for which the quotient $\|M\|_{\ell_{1}^{N}\left(\ell_{\infty}^{K}\right) \otimes_{\min } \ell_{1}^{N}\left(\ell_{\infty}^{K}\right)} /\|M\|_{\ell_{1}^{N}\left(\ell_{\infty}^{K}\right) \otimes_{\epsilon} \ell_{1}^{N}\left(\ell_{\infty}^{K}\right)}$ is large immediately provides an element $\tilde{M}$ for which $\omega^{*}(\tilde{M}) / \omega(\tilde{M})$ is large (see Proposition 2.1 for details). As shown in previous works, connection (1.3) is extremely useful since it allows the use of all the machinery of Banach/operator spaces.

The main tool for proving Theorem 1.1 is a vector-valued version of Schechtman's empirical method. ${ }^{22}$ If $X$ is Banach spaces and $E$ embeds isometrically into $L_{1}(X)$, then $E$ embeds "almost isometrically" into $\ell_{1}^{N}(X)$, with $N$ polynomial in $\operatorname{dim} E$. Our description of $\omega(M)$ and $\omega^{*}(M)$ by means of tensor norms makes the empirical method a natural tool to be applied in our context. However, in order to prove our result we will need to overcome some obstacles. The first one is that the min norm depends on the operator space structure; hence, we will need to deal with an extra matrix structure involved in its definition. The second main difficulty is that the empirical method applies to low dimensional spaces $E$. However, as we will see below, we do not have that property when we deal with the Khot-Vishnoi game. Then, we will need to "cut" the game in order to apply our method. This "cutting" was based on a certain Hilbertian structure of the Khot-Vishnoi game and on the fact that a successful quantum strategy for this game can be constructed with a low dimensional quantum state (we will explain this in more detail in Section IV A).

Our "dimension reduction" result (Proposition 3.1) and its application to operator spaces (in the proof of Proposition 4.2) are quite general and can conceivably be used in other settings. It is worth noting that the Khot-Vishnoi game (a starting point of our investigation) has found numerous applications not only to witness a large deviation of quantum mechanics from classical mechanics but also to show the existence of integrality gaps for some Semidefinite programming (SDP) relaxations of unique games, ${ }^{13}$ and to study new phenomena in quantum information theory such as superactivation of quantum nonlocality. ${ }^{15}$

The paper is organized as follows. In Section II, we will introduce some basic tools about operator spaces and its connections to nonlocal games. In Section III, we will prove a vector-valued version of Schechtman's empirical method, needed for our main theorem. In Section IV, we will first analyze the Khot-Vishnoi game from a mathematical point of view to highlight some of its properties. Then, we will see how the empirical method can be applied on it to obtain Theorem 1.1. 


\section{PRELIMINARIES ON OPERATOR SPACES}

We start by recalling some basic ideas from operator space theory and its connection to nonlocal games; further details can be found in Refs. 7, 21, and 17. An operator space $X$ is a closed subspace of $\mathcal{B}(\mathcal{H})$, the bounded operators on a Hilbert space $\mathcal{H}$. The inclusions $M_{d}(X) \subseteq$ $M_{d}(\mathcal{B}(\mathcal{H})) \simeq \mathcal{B}\left(\mathcal{H}^{\otimes d}\right)$ induce matrix norms $\|\cdot\|_{d}$ on $M_{d}(X)$, where here $M_{d}$ denotes the space of complex matrices of order $d$. Ruan's theorem Ref. 7, [Theorem 2.3.5] states that such matrix norms also characterize operator spaces. More precisely, the existence of such an inclusion into $\mathcal{B}(\mathcal{H})$ is equivalent to having a sequence of matrix norms $\left(M_{d}(X),\|\cdot\|_{d}\right)$ satisfying the following conditions: for every integers $c$ and $d$,

- $\|v \oplus w\|_{c+d}=\max \left\{\|v\|_{c},\|w\|_{d}\right\}$ for every $v \in M_{c}(X)$ and $w \in M_{d}(X)$,

- $\|\alpha \cdot v \cdot \beta\|_{c} \leq\|\alpha\|\|v\|_{c}\|\beta\|$ for every $\alpha, \beta \in M_{c}$ and $v \in M_{c}(X)$.

To specify an operator space structure, one can either provide an explicit inclusion of $X$ into $\mathcal{B}(\mathcal{H})$ or describe the matrix norms on $M_{d}(X)$ for every $d \geq 1$. A canonical example of an operator space is the space of complex matrices of order $N, M_{N}$, with its operator space structure given by the usual identification $M_{N} \simeq \mathcal{B}\left(\mathbb{C}^{N}\right)$. The matrix norm $\|\cdot\|_{d}$ on $M_{d}\left(M_{N}\right)$ is then the usual operator norm on $M_{d N}$. This identification also induces a natural operator space structure on $\ell_{\infty}^{N}$, by identifying this space with the diagonal of $M_{N}$. In particular, given an element $\sum_{i=1}^{N} x_{i} \otimes e_{i} \in M_{d} \otimes \ell_{\infty}^{N}$, the corresponding norm is given by

$$
\left\|\sum_{i=1}^{N} x_{i} \otimes e_{i}\right\|_{M_{d}\left(\ell_{\infty}^{N}\right)}=\sup _{i=1, \ldots, N}\left\|x_{i}\right\|_{M_{N}} .
$$

Given a linear map $T: X \rightarrow Y$ between operator spaces, let $T_{d}:=\operatorname{Id}_{M_{d}} \otimes T: M_{d}(X) \rightarrow M_{d}(Y)$ denote the new linear map defined by

$$
T_{d}\left(\left(v_{i j}\right)_{i, j}\right):=\left(T\left(v_{i j}\right)\right)_{i, j} .
$$

The map $T$ is said to be completely bounded if its completely bounded norm is finite,

$$
\|T\|_{c b}:=\sup _{d \in \mathbb{N}}\left\|T_{d}\right\|<\infty .
$$

In fact, we can define analogously the notion of complete contraction, compete isomorphism, complete isometry, and so on by requiring to have the corresponding property when tensoring with $M_{d}$.

As in the Banach space category, one can define the dual operator space $X^{*}$ of the operator space $X$, with matrix norms given by

$$
M_{d}\left(X^{*}\right)=C B\left(X, M_{d}\right),
$$

where $C B\left(X, M_{d}\right)$ denotes the space of completely bounded maps from $X$ to $M_{d}$ endowed with the completely bounded norm. In particular, this allows us to define an operator space structure on $\ell_{1}^{N}=\left(\ell_{\infty}^{N}\right)^{*}$ and on $S_{1}^{N}=M_{N}^{*}$.

The previous duality relation yields the following: if $\left(e_{i}\right)_{i=1}^{N}$ is the canonical basis of $\ell_{1}^{N}$, and $x_{i}$ are $d \times d$ matrices, then

$$
\left\|\sum_{i} x_{i} \otimes e_{i}\right\|_{M_{d}\left(e_{1}^{N}\right)}=\sup \left\{\left\|\sum_{i} x_{i} \otimes a_{i}\right\|_{d m}: m \in \mathbb{N}, \max _{i}\left\|a_{i}\right\|_{M_{m}} \leq 1\right\} .
$$

Given two operator spaces $X \subseteq \mathcal{B}(\mathcal{H})$ and $Y \subseteq \mathcal{B}(\mathcal{K})$, their algebraic tensor product $X \otimes Y$ is a subspace of $\mathcal{B}\left(\mathcal{H} \otimes \mathcal{K}\right.$ ) and their minimal (or injective) operator space tensor product $X \otimes_{\min } Y$ is the closure of $X \otimes Y$ in $\mathcal{B}(\mathcal{H} \otimes \mathcal{K})$. In particular, note that for every operator space $X$, we trivially obtain that $M_{d} \otimes_{\min } X=M_{d}(X)$ holds isometrically. If $X$ and $Y$ are finite dimensional, then we have a natural algebraic identification $X \otimes Y=L\left(X^{*}, Y\right)$, between the algebraic tensor product and the set of linear maps from $X^{*}$ to $Y$. Here, for a given $u=\sum_{i=1}^{l} x_{i} \otimes y_{i} \in X \otimes Y$, one defines the linear map $T_{u}: X^{*} \rightarrow Y$ by $T_{u}\left(x^{*}\right)=\sum_{i=1}^{l} x^{*}\left(x_{i}\right) y_{i}$ for every $x^{*} \in X^{*}$. In addition, given a linear map $T \in L\left(X^{*}, Y\right)$, we can associate the element $u_{T}=\sum_{i=1}^{s} x_{i} \otimes T\left(x_{i}^{*}\right) \in X \otimes Y$, where $\left(x_{i}\right)_{i=1}^{s}$ and 
$\left(x_{i}^{*}\right)_{i=1}^{s}$ are dual bases of $X$ and $X^{*}$, respectively. The previous correspondence induces the following isometric identifications:

$$
E \otimes_{\epsilon} F=B\left(E^{*}, F\right), \quad X \otimes_{\min } Y=C B\left(X^{*}, Y\right)
$$

for $E$ and $F$ finite dimensional Banach spaces and $X$ and $Y$ finite dimensional operator spaces. We recall that for $u=\sum_{i=1}^{l} e_{i} \otimes f_{i} \in E \otimes F$, its $\epsilon$ norm is defined as

$$
\epsilon(u)=\sup \left\{\left|\sum_{i=1}^{l} e^{*}\left(e_{i}\right) f^{*}\left(f_{i}\right)\right|: e \in B_{E^{*}}, f \in B_{F^{*}}\right\} .
$$

Here, $B_{Z}$ denotes the unit ball of the Banach space $Z$. In analogy, it is not difficult to see that if $u=\sum_{i=1}^{l} x_{i} \otimes y_{i} \in X \otimes Y$,

$$
\|u\|_{\min }=\sup \left\{\left\|\sum_{i=1}^{l} T\left(x_{i}\right) \otimes S\left(y_{i}\right)\right\|_{\mathcal{B}(\mathcal{H} \otimes \mathcal{K})}\right\},
$$

where the supremum runs now over all complete contractions $T: X \rightarrow \mathcal{B}(\mathcal{H}), S: Y \rightarrow \mathcal{B}(\mathcal{K})$.

One can also check that for every operator space $X$, the identification $\ell_{\infty}^{N}(X)=\ell_{\infty}^{N} \otimes_{\min } X$ holds isometrically. This identification allows to endow the Banach space $\ell_{\infty}^{N}(X)$ with a natural operator space structure, which is the one induced by $\ell_{\infty}^{N}(X) \subseteq \ell_{\infty}^{N}(\mathcal{B}(\mathcal{H}))$. According to the comments above, one can naturally use duality (2.1) to obtain an operator space structure on $\ell_{1}^{N}(X)$ for every operator space $X$.

In the current work, we use the space $\ell_{1}^{N}\left(\ell_{\infty}^{K}\right)$, with the norm described below. If $\left(e_{i}\right)_{i=1}^{N}$ and $\left(f_{j}\right)_{j=1}^{K}$ are the canonical bases of $\ell_{1}^{N}$ and $\ell_{\infty}^{K}$, respectively, then, for $d \times d$ matrices $a_{i j}$, we have

$$
\left\|\sum_{i=1}^{N} \sum_{j=1}^{K} a_{i j} \otimes e_{i} \otimes f_{j}\right\|_{M_{d}\left(\ell_{1}^{N}\left(\ell_{\infty}^{K}\right)\right)}=\sup \left\|\sum_{i=1}^{N} \sum_{j=1}^{K} a_{i j} \otimes u_{i}\left(f_{j}\right)\right\|_{M_{d}(B(\mathcal{H}))},
$$

with the supremum taken over all complete contractions $u_{i}: \ell_{\infty}^{K} \rightarrow B(\mathcal{H})\left(u_{i}\right.$ acts on the $i$ th copy of $\ell_{\infty}^{K}$, spanned by $\left.e_{i} \otimes f_{j}, 1 \leq j \leq K\right)$. The dual of $\ell_{1}^{N}\left(\ell_{\infty}^{K}\right)$ is the space $\ell_{\infty}^{N}\left(\ell_{1}^{K}\right)$, whose operator space norm is described, for $d \geq 1$, by

$$
\left\|\sum_{i=1}^{N} \sum_{j=1}^{K} a_{i j} \otimes e_{i} \otimes f_{j}\right\|_{M_{d}\left(e_{\infty}^{N}\left(\ell_{1}^{K}\right)\right)}=\max _{i=1, \ldots, N} \sup \left\{\left\|\sum_{j=1}^{K} a_{i j} \otimes u_{i j}\right\|_{M_{d^{2}}}:\left\|u_{i j}\right\|_{M_{d}} \leq 1\right\} .
$$

Here, $\left(e_{i}\right)_{i=1}^{N}$ and $\left(f_{j}\right)_{j=1}^{K}$ are the canonical bases of $\ell_{\infty}^{N}$ and $\ell_{1}^{K}$, respectively.

\section{A. Connection to nonlocal games}

The following proposition, which was first shown in Ref. 11 and which is explained in much detail in Ref. 17, will be crucial in our work.

Proposition 2.1 (Ref. 11, [Corollary 4]). Let $M=\sum_{x, y, a, b} M_{x, y}^{a, b}\left(e_{x} \otimes e_{a}\right) \otimes\left(e_{y} \otimes e_{b}\right)$ be an element in $\ell_{1}^{N}\left(\ell_{\infty}^{K}\right) \otimes \ell_{1}^{N}\left(\ell_{\infty}^{K}\right)$ such that

$$
\frac{\|M\|_{\ell_{1}^{N}\left(\ell_{\infty}^{K}\right) \otimes_{\min } \ell_{1}^{N}\left(\ell_{\infty}^{K}\right)}}{\|M\|_{\ell_{1}^{N}\left(\ell_{\infty}^{K}\right) \otimes_{\epsilon} \ell_{1}^{N}\left(\ell_{\infty}^{K}\right)}} \geq \alpha .
$$

Then, the Bell functional $\tilde{M}=\left(\tilde{M}_{x, y}^{a, b}\right)_{x, y ; a, b}$, where $x, y=1, \ldots, N$ and $a, b=1, \ldots, K+1, o b$ tained by adding extra zeros to the element $M$ verifies

$$
\frac{\omega^{*}(\tilde{M})}{\omega(\tilde{M})} \geq C \alpha
$$

where $C$ is a universal constant which can be taken equal to $1 / 16$.

In fact, the constant $C$ can be taken $1 / 4$ if one allows to increase the dimension of the corresponding Hilbert space to compute $\omega^{*}(\tilde{M})$, as explained in Ref. 17 [Lemma 4.3]. 
Let us explain here the relation between games and Bell functionals in more detail. This is needed to understand why we are using Bell functionals. On one hand, if we have a game $G$ defined by a predicate function $V$ and a probability distribution $\pi$, it naturally defines a Bell functional by just writing $M_{x, y}^{a, b}=\pi(x, y) V(x, y, a, b)$ for every $x, y, a, b$. Moreover, in this case, the classical and the quantum value of the Bell functional $M$ defined in (1.2) coincide with the classical and the quantum value of the game $G$, respectively. This is the case of the Khot-Vishnoi game $G_{K V}^{n}$ used in Ref. 4 , which actually gives a large quotient $\omega_{n}^{*}\left(G_{K V}^{n}\right) / \omega\left(G_{K V}^{n}\right) \geq C n / \log ^{2} n$.

On the other hand, our construction (given in Section IV) yields a matrix $M$ some of whose coefficients may be negative. Hence, $M$ cannot be a Bell functional given by a nonlocal game $G$. To return to the setting of games (corresponding to matrices with positive coefficients), we observe that for a given Bell functional $M$, we can define the element $G$ as

$$
G_{x, y}^{a, b}=\frac{1}{2 N^{2}}+\frac{1}{2 N^{2} L} M_{x, y}^{a, b}, \quad \text { for every } x, y, a, b,
$$

where $L=\max _{x, y, a, b}\left|M_{x, y}^{a, b}\right|$ and $N$ is the number of questions. It is very easy to check that $G$ has positive coefficients with values in $[0,1]$ and it verifies that

$$
\frac{\beta^{*}(G)}{\beta(G)}=\frac{\omega^{*}(M)}{\omega(M)},
$$

where $\beta(G)$ (resp. $\beta^{*}(G)$ ) is the classical (resp. quantum) bias of the game $G$ defined as

$$
\beta(G)=\sup \left\{|2\langle G, P\rangle-1|: P \in \mathcal{P}_{c}\right\} \quad \text { and } \quad \beta^{*}(G)=\sup \left\{|2\langle G, P\rangle-1|: P \in \mathcal{P}_{q}\right\},
$$

where $\mathcal{P}_{c}$ and $\mathcal{P}_{q}$ denote, respectively, the set of classical and quantum strategies defined in the Introduction.

In particular, given the Khot-Vishnoi game, one can easily find another game, which we will also denote by $G_{K V}^{n}$, for which

$$
\beta_{n}^{*}\left(G_{K V}^{n}\right) / \beta\left(G_{K V}^{n}\right) \geq C n / \log ^{2} n .
$$

This is the game we are referring to in the Introduction right after Equation (1.1).

The previous observation leads to the following consequence.

Proposition 2.2. Let $M=\sum_{x, y, a, b} M_{x, y}^{a, b}\left(e_{x} \otimes e_{a}\right) \otimes\left(e_{y} \otimes e_{b}\right)$ be an element in $\ell_{1}^{N}\left(\ell_{\infty}^{K}\right) \otimes \ell_{1}^{N}$ $\left(\ell_{\infty}^{K}\right)$ such that

$$
\frac{\|M\|_{\ell_{1}^{N}\left(\ell_{\infty}^{K}\right) \otimes_{\min } \ell_{1}^{N}\left(\ell_{\infty}^{K}\right)}}{\|M\|_{\ell_{1}^{N}\left(\ell_{\infty}^{K}\right) \otimes_{\epsilon} \ell_{1}^{N}\left(\ell_{\infty}^{K}\right)}} \geq \alpha .
$$

Then, there exists a game $G$ with $N$ questions and $K+1$ answers, with $\frac{\beta^{*}(G)}{\beta(G)} \geq C \alpha$, where, as before, we can take $C$ to be $1 / 16$.

\section{B. The operator space $\mathrm{OH}$}

In order to present a self-contained work, we will introduce in this section some basic definitions and results about the so-called $O H$-space.

In this paper, we use the notion of the conjugate space. If $X$ is an operator space, then $\bar{X}$ is the same space but with conjugate multiplication. More specifically, denote by $\bar{x}$ the element of $x \in X$ when considered as sitting in $\bar{X}$. Then $\bar{\lambda} \cdot \bar{x}=\overline{\lambda x}$. Thus, the map $X \rightarrow \bar{X}: x \mapsto \bar{x}$ is an antilinear isometry. If $X \subseteq \mathcal{B}(\mathcal{H})$, its conjugate operator space structure is given by the embedding $\bar{X} \subseteq \overline{\mathcal{B}(\mathcal{H})}=\mathcal{B}(\overline{\mathcal{H}})$. We can therefore describe the operator space structure on $\bar{X}$ via

$$
\left\|\sum_{i} a_{i} \otimes \overline{x_{i}}\right\|_{M_{d}(\bar{X})}=\left\|\sum_{i} \overline{a_{i}} \otimes x_{i}\right\|_{M_{d}(X)} \quad \text { for every } d .
$$

If $X$ consists of matrices $\left(a_{i j}\right)_{i, j=1}^{\infty} \in B\left(\ell_{2}\right)$ (with respect to a certain basis of $\ell_{2}$ ), then we can view elements of $\bar{X}$ as matrices $\left(\overline{a_{i j}}\right)$. We refer the reader to Ref. 21 [Section 2.9] for more information. 
In general, the formal identity $X \rightarrow \bar{X}$ need not be a complete isometry. However, for the case we are interested in, $X=\ell_{\infty}^{N}\left(\ell_{1}^{K}\right)$, the formal identification of bases yields a linear complete isometry. Indeed, let $\left(e_{i}\right)_{i=1}^{N}$ and $\left(f_{j}\right)_{j=1}^{K}$ be the bases of $\ell_{\infty}^{N}$ and $\ell_{1}^{K}$, respectively, then $e_{i} \otimes f_{j}$ is the "canonical" basis of $X$. For $d \times d$ matrices $a_{i j},(2.3)$ gives

$$
\begin{aligned}
& \left\|\sum_{i=1}^{N} \sum_{i=1}^{K} a_{i j} \otimes e_{i} \otimes f_{j}\right\|_{M_{d}(X)}=\max _{i=1, \ldots, N} \sup \left\{\left\|\sum_{j=1}^{K} a_{i j} \otimes \overline{u_{i j}}\right\|_{M_{d^{2}}}:\left\|u_{i j}\right\|_{M_{N}} \leq 1\right\} \\
& =\max _{i=1, \ldots, N} \sup \left\{\left\|\sum_{j=1}^{K} \overline{a_{i j}} \otimes u_{i j}\right\|_{M_{d^{2}}}:\left\|u_{i j}\right\|_{M_{d}} \leq 1\right\}=\left\|\sum_{i=1}^{N} \sum_{i=1}^{K} \overline{a_{i j}} \otimes e_{i} \otimes f_{j}\right\|_{M_{d}(X)} \\
& =\left\|\sum_{i=1}^{N} \sum_{i=1}^{K} a_{i j} \otimes \overline{e_{i} \otimes f_{j}}\right\|_{M_{d}(\bar{X})}
\end{aligned}
$$

A similar computation shows that the basis $\left(e_{i} \otimes f_{j}\right)$ is 1-completely unconditional; that is,

$$
\left\|\sum_{i=1}^{N} \sum_{i=1}^{K} a_{i j} \otimes \alpha_{i j} e_{i} \otimes f_{j}\right\|_{M_{d}(X)}=\left\|\sum_{i=1}^{N} \sum_{i=1}^{K} a_{i j} \otimes e_{i} \otimes f_{j}\right\|_{M_{d}(X)}
$$

whenever $a_{i j} \in M_{d}$ and $\left|\alpha_{i j}\right|=1$ for any $i, j$.

As $(\bar{X})^{*}=\overline{X^{*}}$ (with conjugate action $\left\langle\overline{x^{*}}, \bar{x}\right\rangle=\overline{\left\langle x^{*}, x\right\rangle}$ ), the two preceding statements hold for the space $X=\ell_{1}^{N}\left(\ell_{\infty}^{K}\right)$ (the dual of $\left.\ell_{\infty}^{N}\left(\ell_{1}^{K}\right)\right)$ as well. More precisely, if $a_{i j} \in M_{d}$ and $\left|\alpha_{i j}\right|=1$ for any $(i, j)$, then

$$
\left\|\sum_{i=1}^{N} \sum_{i=1}^{K} a_{i j} \otimes \alpha_{i j} e_{i} \otimes f_{j}\right\|_{M_{d}(X)}=\left\|\sum_{i=1}^{N} \sum_{i=1}^{K} a_{i j} \otimes e_{i} \otimes f_{j}\right\|_{M_{d}(X)}=\left\|\sum_{i=1}^{N} \sum_{i=1}^{K} a_{i j} \otimes \overline{e_{i} \otimes f_{j}}\right\|_{M_{d}(\bar{X})} .
$$

Consequently, suppose $y_{k} \in \ell_{1}^{N}\left(\ell_{\infty}^{K}\right)$ are of the form $y_{k}=\sum_{i, j} \alpha_{i j} e_{i} \otimes f_{j}$, where $\alpha_{i j}$ are real numbers. Then, for all $a_{k} \in M_{d}$, we have

$$
\left\|\sum_{k=1}^{M} a_{k} \otimes y_{k}\right\|_{M_{d}\left(\ell_{1}^{N}\left(\ell_{\infty}^{K}\right)\right)}=\left\|\sum_{k=1}^{M} a_{k} \otimes \overline{y_{k}}\right\|_{M_{d}\left(\overline{\ell_{1}^{N}\left(\ell_{\infty}^{K}\right)}\right)} .
$$

In a similar fashion, we can show that

$$
\left\|\sum_{k=1}^{M} a_{k} \otimes y_{k}\right\|_{M_{d} \otimes_{\epsilon} \ell_{1}^{N}\left(\ell_{\infty}^{K}\right)}=\left\|\sum_{k=1}^{M} a_{k} \otimes \overline{y_{k}}\right\|_{M_{d} \otimes_{\epsilon} \overline{\ell_{1}^{N}\left(\ell_{\infty}^{K}\right)}} .
$$

We also use the operator Hilbert space $\mathrm{OH}_{N}$, introduced by Pisier in Ref. 19. On the Banach space level, it is the space $\ell_{2}^{N}$ with matrix norms given by

$$
\left\|\sum_{i=1}^{N} x_{i} \otimes e_{i}\right\|_{M_{d}\left(O H_{N}\right)}=\left\|\sum_{i=1}^{N} x_{i} \otimes \overline{x_{i}}\right\|_{M_{d}\left(\overline{M_{d}}\right)}^{1 / 2},
$$

where $\left(e_{i}\right)_{i=1}^{N}$ is an orthonormal basis of $\ell_{2}^{N}$. In light of the above discussion on complex conjugation, we can view $\overline{x_{i}}$ as obtained from $x_{i}$ by entrywise complex conjugation and $\sum_{i=1}^{N} x_{i} \otimes \overline{x_{i}}$ as a $d^{2} \times d^{2}$ matrix. Hence, if each matrix $x_{i}$ has real entries (in a certain basis), then

$$
\left\|\sum_{i=1}^{N} x_{i} \otimes e_{i}\right\|_{M_{d}\left(O H_{N}\right)}=\left\|\sum_{i=1}^{N} x_{i} \otimes x_{i}\right\|_{M_{d^{2}}} .
$$

It is easy to check from its definition that the operator space $O H_{N}$ is homogeneous; meaning that for every linear operator $T: \ell_{2}^{N} \rightarrow \ell_{2}^{K}$ the following equality holds:

$$
\left\|T: \ell_{2}^{N} \rightarrow \ell_{2}^{K}\right\|=\left\|T: O H_{N} \rightarrow O H_{K}\right\|_{c b} .
$$

One can check that the canonical isometric identification $\ell_{2}^{N} \simeq \overline{\left(\ell_{2}^{N}\right)^{*}}$ at the Banach space level induces a complete isometry from $O H_{N}$ to $\overline{O H_{N}^{*}}$. In fact, $O H_{N}$ is the unique operator space with this property, up to complete isometries. 
Let us consider a linear map $v: \ell_{2}^{N} \rightarrow X$, where $X$ is a Banach space. Let us also fix an orthonormal basis $\left(\theta_{i}\right)_{i=1}^{N}$ of $\ell_{2}^{N}$. Then, it is very easy to see that

$$
\|v\|^{2}=\sup \left\{\sum_{i=1}^{N}\left|\left\langle f, v \theta_{i}\right\rangle\right|^{2}: f \in B_{X^{*}}\right\} .
$$

By the definition of the injective tensor product of Banach spaces,

$$
\begin{aligned}
\left\|\sum_{i=1}^{N} v\left(\theta_{i}\right) \otimes \overline{v\left(\theta_{i}\right)}\right\|_{X \otimes_{\epsilon} \bar{X}} & =\sup \left\{\left|\sum_{i=1}^{N}\left\langle f, v \theta_{i}\right\rangle\left\langle\bar{g}, \overline{v \theta_{i}}\right\rangle\right|: f, g \in B_{X^{*}}\right\} \\
& =\sup \left\{\left|\sum_{i=1}^{N}\left\langle f, v \theta_{i}\right\rangle \overline{\left\langle g, v \theta_{i}\right\rangle}\right|: f, g \in B_{X^{*}}\right\} .
\end{aligned}
$$

Combining Hölder's inequality with (2.7), we obtain

$$
\left\|\sum_{i=1}^{N} v\left(\theta_{i}\right) \otimes \overline{v\left(\theta_{i}\right)}\right\|_{X \otimes_{\epsilon} \bar{X}} \leq \sup \left\{\sum_{i=1}^{N}\left|\left\langle f, v \theta_{i}\right\rangle\right|^{2}: f \in B_{X^{*}}\right\} \leq\|v\|^{2} .
$$

Moreover, plugging $f=g$, for which the supremum in (2.7) is attained, into (2.8), we show that

$$
\|v\|^{2}=\left\|\sum_{i=1}^{N} v\left(\theta_{i}\right) \otimes \overline{v\left(\theta_{i}\right)}\right\|_{X \otimes_{\epsilon} \bar{X}}
$$

The previous paragraph proves the first point of the following proposition. The proof of the second part can be found in Ref. 21 [Proposition 7.2].

Proposition 2.3. Suppose $\left(\theta_{i}\right)_{i=1}^{N}$ is an orthonormal basis in $\mathrm{OH}_{N}, X$ is an operator space, and $v: \mathrm{OH}_{N} \rightarrow X$ is a linear map. Then,

(2) $\|v\|_{c b}^{2}=\left\|\sum_{i=1}^{N} v\left(\theta_{i}\right) \otimes \overline{v\left(\theta_{i}\right)}\right\|_{X \otimes_{\min } \bar{X}}$.

The following version of the preceding result follows directly from the earlier discussion on complex conjugation.

Corollary 2.4. Suppose $\left(\theta_{i}\right)_{i=1}^{N}$ is an orthonormal basis in $O H_{N}$ and $v: O H_{N} \rightarrow M_{d}$ is a linear map, so that for any $1 \leq i \leq N, v\left(\theta_{i}\right)$ is a real linear combination of matrix units. Then,

$$
\|v\|^{2}=\left\|\sum_{i=1}^{N} v\left(\theta_{i}\right) \otimes v\left(\theta_{i}\right)\right\|_{M_{d^{2}}} .
$$

Here and below, the word "matrix units (in $M_{d}$ )" refers to $d \times d$ matrices, in which 1 entry is 1 and other entries vanish (with respect to a certain fixed orthonormal basis of $\ell_{2}^{n}$ ). Clearly the matrix units form a basis for $M_{d}$.

\section{VECTOR-VALUED EMPIRICAL METHOD}

In this section, we prove a vector-valued version of Schechtman's empirical method. ${ }^{22}$

Proposition 3.1. Let $E$ be an m-dimensional subspace of $L_{r}(\mu, X)$, where $X$ is a Banach space, $(\Omega, \mu)$ is a probability space, and $1 \leq r<\infty$. For any given $\epsilon \in(0,1 / 2)$ there exists a constant $C(\varepsilon)$ such that if we consider $n=\left\lceil C(\varepsilon) m^{1+r}\right\rceil$, then $\ell_{r}^{n}(X)$ contains a subspace $E^{\prime}$ which is $(1+\varepsilon)$-isomorphic to $E$. We can take $C(\varepsilon)=C_{0} \varepsilon^{-2} \log \left(\varepsilon^{-1}\right)$ for a universal constant $C_{0}$. 
We will first prove an easy lemma which will make the proof of Proposition 3.1 simpler. To this end, let us consider a normalized Auerbach basis $\left(e_{i}\right)_{i=1}^{m}$ in $E$. Note that we clearly have $\left\|e_{i}\right\|_{E}^{r}=\int_{\Omega}\left\|e_{i}(t)\right\|_{X}^{r}$ $d \mu(t)=1$ for every $i=1, \ldots, m$. Let us define the function $\phi(t)=m^{-1} \sum_{i=1}^{m}\left\|e_{i}(t)\right\|_{X}^{r}$ for every $t \in \Omega$. It is then obvious that $\int_{0}^{1} \phi(t) d \mu(t)=1$ so that $d v=\phi d \mu$ defines a probability measure on $\Omega$.

Lemma 3.2. Let us define the linear map $S: E \rightarrow L_{r}(v, X)$ by $S(e)=\phi^{-1 / r}$ e for every $e \in E$ with the convention $\frac{0}{0}=0$. Then, $S$ is an isometry and, moreover, for any $e \in E$ we have

$$
\|S e\|_{L_{\infty}(v, X)} \leq m\|e\|_{L_{r}(\mu, X)}=m\|S e\|_{L_{r}(v, X)} .
$$

Proof. Note that if $\phi(t)=0$ for some $t$, then $e(t)=0$ for every $e \in E$. Thus, $S$ is well defined. The linearity of $S$ is obvious. Now, for any given $e \in E$ we have

$$
\|S e\|_{L_{r}(v, X)}^{r}=\int_{\Omega}\left\|\phi^{-1 / r}(t) e(t)\right\|_{X}^{r} \phi(t) d \mu(t)=\|e\|_{L_{r}(\mu, X)}^{r} .
$$

Hence, $S$ is indeed an isometry. Finally, given a norm one element $e=\sum_{i=1}^{m} \alpha_{i} e_{i} \in E$, the fact that $\left(e_{i}\right)_{i=1}^{m}$ is an Auerbach basis implies that $\max _{i}\left|\alpha_{i}\right| \leq 1$. Hence,

$$
\|(S e)(t)\|_{X}=\phi^{-1 / r}(t)\|e(t)\|_{X}=m^{1 / r}\left(\sum_{i=1}^{m}\left\|e_{i}(t)\right\|_{X}^{r}\right)^{-1 / r}\left\|\sum_{i=1}^{m} \alpha_{i} e_{i}(t)\right\|_{X} .
$$

Let $r^{\prime}=\frac{r}{r-1}$ so that $\frac{1}{r}+\frac{1}{r^{\prime}}=1$. By Hölder's inequality,

$$
\left\|\sum_{i=1}^{m} \alpha_{i} e_{i}(t)\right\|_{X} \leq\left(\sum_{i=1}^{m}\left|\alpha_{i}\right|^{r^{\prime}}\right)^{1 / r^{\prime}}\left(\sum_{i=1}^{m}\left\|e_{i}(t)\right\|_{X}^{r}\right)^{1 / r} \leq m^{1 / r^{\prime}}\left(\sum_{i=1}^{m}\left\|e_{i}(t)\right\|_{X}^{r}\right)^{1 / r} .
$$

Hence, for almost every $t$,

$$
\|(S e)(t)\|_{X} \leq m^{1 / r}\left(\sum_{i=1}^{m}\left\|e_{i}(t)\right\|_{X}^{r}\right)^{-1 / r} m^{1 / r^{\prime}}\left(\sum_{i=1}^{m}\left\|e_{i}(t)\right\|_{X}^{r}\right)^{1 / r}=m .
$$

The following lemma is a standard large deviation inequality for sums of independent random variables. The proof can be found in Ref. 22 [Lemma 3].

Lemma 3.3. Let $\left(y_{i}\right)_{i=1}^{n}$ be a family of independent random variables and let $A$ and $B$ be non-negative constants such that $\mathbb{E} y_{i}=0, \mathbb{E}\left|y_{i}\right| \leq A$, and $\left\|y_{i}\right\|_{\infty} \leq B$ for every $i=1, \ldots, n$. Then,

$$
\mathbb{P}\left(\left|\sum_{i=1}^{n} y_{i}\right|>c\right) \leq 2 \exp \left(-\frac{c^{2}}{4 e A B n}\right)
$$

for all $c \leq 2 e A n$.

For the proof of Proposition 3.1 we use some ideas from Ref. 3 [Section 2] and from Ref. 22. We recall first that for a given Banach space $E$, an $\eta$-net in the unit sphere of $E$ is a set of points $\mathcal{N}$ in the unit sphere so that for any point $e$ in the sphere there exists an $x \in \mathcal{N}$ such that $\|x-e\| \leq \eta$.

Proof of Proposition 3.1. According to Lemma 3.2 we can assume that $\|e\|_{L_{\infty}(\mu, X)} \leq$ $m\|e\|_{L_{r}(\mu, X)}$, for any $e \in E$. On the other hand, for every $t=\left(t_{1}, \ldots, t_{n}\right) \in \Omega^{n}$, we consider the linear map $T_{t}: L_{r}(\mu, X) \rightarrow L_{r}^{n}(X)$ defined by $T_{t}(f)=\left(f\left(t_{i}\right)\right)_{i=1}^{n}$. Here, $L_{r}^{n}$ denotes the space $\mathbb{R}^{n}$ endowed with the norm $\left\|\left(\alpha_{i}\right)_{i=1}^{n}\right\|_{L_{r}^{n}}^{r}=n^{-1} \sum_{i=1}^{n}\left|\alpha_{i}\right|^{r}$.

Now, let $e \in E$ be any fixed element such that $\|e\|_{E}=1$. For $1 \leq i \leq n$ we consider the random variable $y_{i}: \Omega^{n} \rightarrow \mathbb{R}$ defined by $y_{i}(t)=\left\|e\left(t_{i}\right)\right\|_{X}^{r}-1$ for every $t$. Then, $\left(y_{i}\right)_{i=1}^{n}$ is a family of independent random variables satisfying $\mathbb{E} y_{i}=0, \mathbb{E}\left|y_{i}\right| \leq 2$, and $\left\|y_{i}\right\|_{\infty} \leq m^{r}$ for every $1 \leq i \leq n$. On the other hand, $\left\|T_{t}(e)\right\|_{L_{r}^{n}(X)}^{r}-1=n^{-1} \sum_{i=1}^{n} y_{i}(t)$ for every $t \in \Omega^{n}$. According to Lemma 3.3 it follows that

$$
\mathbb{P}\left(t \in \Omega^{n}:\left|\left\|T_{t}(e)\right\|_{r}^{r}-1\right| \geq c\right)=\mathbb{P}\left(t \in \Omega^{n}:\left|\sum_{i=1}^{n} y_{i}(t)\right| \geq c n\right) \leq 2 \exp \left(-\frac{c^{2} n}{8 e m^{r}}\right)
$$

for any $c \in(0,1)$. Note that if $\left|\left\|T_{t}(e)\right\|_{L_{r}^{n}(X)}^{r}-1\right| \leq c$, then $1-c \leq\left\|T_{t}(e)\right\|_{L_{r}^{n}(X)} \leq 1+c$. 
Let $\eta=\varepsilon / 4$. Standard techniques allow us to find an $\eta$-net $\mathcal{N}$ in the unit sphere of $E$, with $|\mathcal{N}| \leq\left(\frac{3}{\eta}\right)^{m}=\left(\frac{12}{\epsilon}\right)^{m}$. If we consider the particular choice $c=\eta$, we have

$$
2 \exp \left(-\frac{\eta^{2} n}{8 e m^{r}}\right)|\mathcal{N}| \leq 2 \exp \left(m \log \frac{12}{\epsilon}-\frac{\eta^{2} n}{8 e m^{r}}\right)<1,
$$

where the last inequality follows from our choice $n=\left\lceil C(\varepsilon) m^{1+r}\right\rceil$ with $C(\varepsilon)=C_{0} \varepsilon^{-2} \log \left(\varepsilon^{-1}\right)$ for a certain universal constant $C_{0}$. This means that there is a strictly positive probability of having an element $t \in \Omega^{n}$ such that $1-\eta \leq\left\|T_{t}(e)\right\|_{L_{r}^{n}(X)} \leq 1+\eta$ for every $e \in \mathcal{N}$. We claim that

$$
1-\varepsilon \leq\left\|T_{t}(e)\right\|_{L_{r}^{n}(X)} \leq 1+\varepsilon
$$

for every $e$ in the unit sphere of $E$. If this is so, we conclude the proof by noting that the map $k: L_{r}^{n}(X) \rightarrow \ell_{r}^{n}(X)$ defined by $k\left(\left(x_{i}\right)_{i=1}^{n}\right)=n^{-\frac{1}{r}}\left(x_{i}\right)_{i=1}^{n}$ is an isometry.

Note first that such an $e$ can be written as $e=e_{0}+\sum_{n=1}^{\infty} a_{n} e_{n}$, with $e_{n} \in \mathcal{N}$ and $\left|a_{n}\right| \leq \eta^{n}$ for every $n \geq 0$. This can be done by an iterative process. Given the norm one element $e$, there exists an element $e_{0} \in \mathcal{N}$ such that $\left\|e_{0}-e\right\| \leq \delta$. Hence, we can write $e=e_{0}+a_{1} e_{0}^{\prime}$ with $\left|a_{1}\right| \leq \delta$ and $\left\|e_{0}^{\prime}\right\|=1$. Now, given such an element $e_{0}^{\prime}$, there must exist $e_{1} \in \mathcal{N}$ so that $\left\|e_{0}^{\prime}-e_{1}\right\| \leq \delta$. Hence, $e_{0}^{\prime}=e_{1}+a_{1}^{\prime} e_{1}^{\prime}$ with $\left|a_{1}^{\prime}\right| \leq \delta$ and $\left\|e_{1}^{\prime}\right\|=1$. We can then write $e=e_{0}+a_{1} e_{1}+a_{1} a_{1}^{\prime} e_{1}^{\prime}$. The iteration of this process leads to a sum $e=e_{0}+\sum_{n=1}^{\infty} a_{n} e_{n}$ as stated above.

By the triangle inequality,

$$
\left\|T_{t}(e)\right\| \leq\left\|T_{t}\left(e_{0}\right)\right\|+\sum_{k=1}^{\infty}\left|a_{n}\right|\left\|T_{t}\left(e_{n}\right)\right\| \leq(1+\eta) \sum_{k=0}^{\infty} \eta^{k}=\frac{1+\eta}{1-\eta}<1+\epsilon .
$$

On the other hand,

$$
\left\|T_{t}(e)\right\| \geq\left\|T_{t}\left(e_{0}\right)\right\|-\sum_{k=1}^{\infty}\left|a_{n}\right||| T_{t}\left(e_{n}\right) \| \geq(1-\eta)-(1+\eta) \sum_{k=1}^{\infty} \eta^{k}=1-\eta-\frac{\eta(1+\eta)}{1-\eta}>1-\epsilon .
$$

Together, the two centered inequalities imply (3.1).

Remark 3.1. In this work, we will be mostly interested in the space $\ell_{1}^{N}(X)$. Let us assume that we have an m-dimensional subspace $E \subset \ell_{1}^{N}(X)$. Proposition 3.1 tells us that if we consider $n=$ $\left\lceil C(\varepsilon) m^{2}\right\rceil$, then there exists a map $J: \ell_{1}^{N} \rightarrow \ell_{1}^{n}$ defined by some indices $i_{1}, \ldots, i_{n} \in\{1, \ldots, N\}$ and some positive numbers $\alpha_{1}, \ldots, \alpha_{n}$ such that $J\left(x_{1}, \ldots, x_{N}\right)=\left(\alpha_{1} x_{i_{1}}, \ldots, \alpha_{n} x_{i_{n}}\right)$ for every $\left(x_{1}, \ldots\right.$, $\left.x_{N}\right) \in \ell_{1}^{N}$ and such that $J \otimes i d_{X}$ defines a $(1+\epsilon)$-isomorphism from $E$ to $\ell_{1}^{n}(X)$.

\section{MAIN RESULT}

In this section, we will prove our main theorem. We will start by re-proving the classical and the quantum bounds for the Khot-Vishnoi game in the language of operator spaces. In particular, we show that this game can be understood as a map factorizing through a Hilbert space. This fact will be crucial in our analysis later.

Our results deal with operators from $O H_{N}$ into other spaces. To recast the results in more familiar terms, we introduce some notation. Suppose $X$ and $Y$ are normed spaces with bases $\left(x_{i}\right)$ and $\left(y_{j}\right)$, respectively. For any $v: X \rightarrow Y$, there exists a unique family $\left(\alpha_{i j}\right)$ so that $v x_{i}=\sum_{j} \alpha_{i j} y_{j}$. We say that $v$ is real (resp. positive) with respect to these bases if, for any $i$ and $j$, we have $\alpha_{i j} \in \mathbb{R}$ (resp. $\alpha_{i j} \geq 0$ ). We often refer to the "canonical" basis of $\ell_{1}^{N}\left(\ell_{\infty}^{n}\right)$ as the one consisting in elements $e_{i} \otimes f_{j}$, while that of $M_{d}$ - of matrix units.

Proposition 4.1. Suppose $n$ is a power of 2, and let $N=\frac{2^{n}}{n}$. Then there exists an operator $V: O H_{N n} \rightarrow \ell_{1}^{N}\left(\ell_{\infty}^{n}\right)$ and a completely positive complete contraction $U: \ell_{1}^{N}\left(\ell_{\infty}^{n}\right) \rightarrow M_{n}$ so that

$$
\|U V\|_{c b}=\Omega\left(\frac{\sqrt{N}}{\log n}\right) \text { and }\|V\|=O\left(\sqrt{\frac{N}{n}}\right) .
$$

Moreover, $V$ is positive with respect to the canonical bases of its domain and range, and $U$ is real with respect to the canonical bases. 
Denote by $\left(\delta_{x}\right)_{x \in\{0,1\}^{n}}$ the canonical orthonormal basis of $\ell_{2}^{N n}=\ell_{2}\left(\{0,1\}^{n}\right)$. Let

$$
M=\sum_{x} V \delta_{x} \otimes V \delta_{x} \in \ell_{1}^{N}\left(\ell_{\infty}^{n}\right) \otimes \ell_{1}^{N}\left(\ell_{\infty}^{n}\right)
$$

By Proposition 2.3 and Corollary 2.4, (2.4), and (2.5), $\|M\|_{\epsilon}=\|V\|^{2}$ and $\|M\|_{\min }=\|V\|_{c b}^{2}$. The interested reader can check that the tensor $M$ has the form $N G_{K V}$, where $G_{K V}$ denotes the Khot-Vishnoi game (see Ref. 4 [Section 4] for a precise description; in particular, $M$ has positive entries).

Note that in Proposition 4.1 and the remark following it, the operators $U$ and $V$, and the tensor product $M$, depend on $n$.

Proof of Proposition 4.1. Consider the Cantor group $G=\{0,1\}^{n}$, and let $G_{0}$ be its Hadamard subgroup, of cardinality $n$. Let $\Omega=G / G_{0}$ (then $|\Omega|=N$ ). It is important to note (for future reference) that for any distinct $x, y \in G_{0}, d(x, y)=n / 2$, where here $d(\cdot, \cdot)$ stands for the Hamming metric. Consider the probability measures

$$
\mu_{0}=\frac{1+\sqrt{\varepsilon}}{2} \delta_{0}+\frac{1-\sqrt{\varepsilon}}{2} \delta_{1} \text { and } \mu=\mu_{0}^{\otimes n},
$$

on $\{0,1\}$ and $G$, respectively (the number $\varepsilon \in(0,1)$ will be specified later). The operator $V: \mathbb{C}^{G} \rightarrow$ $\mathbb{C}^{G}$ is defined as the convolution with the measure $\mu: V f=C_{\mu} f=f * \mu$, or in other words,

$$
[V f](x)=\sum_{y \in G}\left(\frac{1+\sqrt{\varepsilon}}{2}\right)^{n-d(x, y)}\left(\frac{1-\sqrt{\varepsilon}}{2}\right)^{d(x, y)} f(y)
$$

(all entries of the matrix representing $V$ are positive). Given any set $A \subset\{1,2, \ldots, n\}$, let us denote the Walsh function $w_{A}:\{0,1\}^{n} \rightarrow \mathbb{R}$, defined by $w_{A}(x)=(-1)^{\sum_{i \in A} x_{i}}$. It is easy to see that the Walsh functions are eigenvectors of $V: V w_{A}=\varepsilon^{|A| / 2} w_{A}$. For other properties of this operator, see, e.g., Ref. 23. We view $V$ as acting from $O H_{N n}$ to $\ell_{1}^{N}\left(\ell_{\infty}^{n}\right)$. The identification of $O H_{N n}$ with $\ell_{2}(G)$ is straightforward. For the identification with $\ell_{1}^{N}\left(\ell_{\infty}^{n}\right)$, given $g \in G$, we identify $\delta_{g}$ with $\delta_{[g]} \otimes \Phi_{[g]}(g)$, where for every $[g], \Phi_{[g]}:[g] \rightarrow[n]$ defines a fixed enumeration of the elements in the class $[g]$.

Using the techniques of Ref. 4, we prove that $\|V\| \prec \sqrt{N} / \sqrt{n}$ and $\|U V\|_{c b}>\sqrt{N} / \log n$, where here we use symbols $<$ and $>$ to denote inequality up to universal constants independent of the dimension. Indeed, consider the factorization $V=i_{p} C_{\mu} j_{2}$, where $j_{2}$ is the formal identity from $\ell_{2}^{N n}$ to $L_{2}^{N n}=L_{2}(G)$, where this last space is equipped with the uniform probability measure on $G, C_{\mu}: L_{2}(G) \rightarrow L_{p}(G)$ is the convolution with $\mu, p=(1+\varepsilon) / \varepsilon$, and $i_{p}: L_{p}(G) \rightarrow \ell_{1}^{N}\left(\ell_{\infty}^{n}\right)$ is the formal identity. We clearly have $\left\|j_{2}\right\|=(N n)^{-1 / 2}$. Furthermore, $\left\|i_{p}\right\|=N n^{1 / p}$. Indeed, this follows by noting that

$$
\left\|i_{p}\right\| \leq\left\|i_{p}: L_{p}(G) \rightarrow \ell_{p}^{N}\left(\ell_{p}^{n}\right)\right\|\left\|i d: \ell_{p}^{N}\left(\ell_{p}^{n}\right) \rightarrow \ell_{1}^{N}\left(\ell_{\infty}^{n}\right)\right\| \leq(N n)^{1 / p} N^{1-1 / p}=N n^{1 / p} .
$$

Finally, $p$ is selected to make $C_{\mu}$ contractive, by Bonami-Beckner hypercontractivity inequality (see, e.g., Ref. 23, [Theorem 4.1]). This gives

$$
\|V\| \leq\left\|i_{p}\right\|\left\|C_{\mu}\right\|\left\|j_{2}\right\|=N n^{1 / p}(N n)^{-1 / 2}=N^{1 / 2} n^{1 / p-1 / 2}=N^{1 / 2} n^{\varepsilon /(1+\varepsilon)-1 / 2} .
$$

To define the operator $U$, consider, for each $x=\left(x_{1}, \ldots, x_{n}\right) \in G=\{0,1\}^{n}$, the unit vector

$$
h_{x}=\frac{1}{\sqrt{n}} \sum_{i=1}^{n}(-1)^{x_{i}} e_{i} \in \ell_{2}^{n} .
$$

Here, $e_{1}, \ldots, e_{n}$ are the elements of the canonical basis in $\ell_{2}^{n}$. Let $p_{x}=h_{x} \otimes h_{x} \in M_{n}$ be the orthogonal projection onto $\mathbb{C} h_{x}$, and define $U: \ell_{1}^{N}\left(\ell_{\infty}^{n}\right) \rightarrow M_{n}: \delta_{x} \mapsto p_{x}$. As noted in Ref. 4, if $x, y \in G$ are distinct and belong to the same coset of $\Omega=G / G_{0}$, then $d(x, y)=n / 2$, hence $\left\langle h_{x}, h_{y}\right\rangle=0$, and consequently, $p_{x} p_{y}=0$. Therefore, the restriction of $U$ to any copy of $\ell_{\infty}^{n}$ in its domain is a complete isometry. Thus, $U$ is a complete contraction. Moreover, $U \delta_{x}$ has real entries for any $x$, hence $U$ is represented by a real matrix (relative to canonical bases).

By construction, $U$ is real with respect to the standard bases. Combining Proposition 2.3 with (2.4), we obtain $\|U V\|_{c b}^{2}=\left\|\sum_{x \in G} U V \delta_{x} \otimes U V \delta_{x}\right\|_{M_{n} \otimes_{\min } M_{n}}$. Identify $M_{n} \otimes_{\min } M_{n}$ with $M_{n^{2}}$ and 
consider the maximally entangled state, defined as $f(A)=\langle A \xi, \xi\rangle$ for every $A \in M_{n^{2}}$, where

$$
\xi=\frac{1}{\sqrt{n}} \sum_{i=1}^{n} e_{i} \otimes e_{i}
$$

For $a, b \in M_{n}, f(a \otimes b)=\frac{1}{n} \sum_{i, j=1}^{n}\left\langle e_{j}, a e_{i}\right\rangle\left\langle e_{j}, b e_{i}\right\rangle=\frac{1}{n} \operatorname{tr}\left(a b^{t r}\right)$. Thus,

$$
f\left(p_{x} \otimes p_{y}\right)=\frac{1}{n}\left\langle h_{x}, h_{y}\right\rangle^{2}=\frac{n-2 d(x, y)}{n^{2}} .
$$

As $V=V^{*}, \sum_{x \in G} V \delta_{x} \otimes V \delta_{x}$ can be identified on the vector space level with the operator $C_{\mu}^{2}: \mathbb{C}^{G} \rightarrow \mathbb{C}^{G}$. For any Walsh function $w_{A}$, we have $C_{\mu}^{2} w_{A}=\varepsilon^{|A|} w_{A}$, hence $C_{\mu}^{2}=C_{\nu}$, for the measure $v=\left(\frac{1+\varepsilon}{2} \delta_{0}+\frac{1-\varepsilon}{2} \delta_{1}\right)^{\otimes n}$. Therefore,

$$
\sum_{x \in G} V \delta_{x} \otimes V \delta_{x}=\sum_{y, z \in G}\left(\frac{1+\varepsilon}{2}\right)^{n-d(y, z)}\left(\frac{1-\varepsilon}{2}\right)^{d(y, z)} \delta_{y} \otimes \delta_{z},
$$

yielding

$$
\sum_{x \in G} U V \delta_{x} \otimes U V \delta_{x}=\sum_{y, z \in G}\left(\frac{1+\varepsilon}{2}\right)^{n-d(y, z)}\left(\frac{1-\varepsilon}{2}\right)^{d(y, z)} p_{y} \otimes p_{z} .
$$

Consequently,

$$
\begin{aligned}
\|U V\|_{c b}^{2}= & \left\|\sum_{x \in G} U V \delta_{x} \otimes U V \delta_{x}\right\| \geq \sum_{y, z \in G}\left(\frac{1+\varepsilon}{2}\right)^{n-d(y, z)}\left(\frac{1-\varepsilon}{2}\right)^{d(y, z)} f\left(p_{y} \otimes p_{z}\right) \\
& =\frac{1}{n} \sum_{y, z \in G}\left(\frac{1+\varepsilon}{2}\right)^{n-d(y, z)}\left(\frac{1-\varepsilon}{2}\right)^{d(y, z)}\left(1-2 \frac{d(z, y)}{n}\right)^{2} \\
& =N \sum_{a \in G}\left(\frac{1+\varepsilon}{2}\right)^{n-|a|}\left(\frac{1-\varepsilon}{2}\right)^{|a|}\left(1-2 \frac{|a|}{n}\right)^{2}=N \mathbb{E}\left(1-2 \frac{X}{n}\right)^{2},
\end{aligned}
$$

where $X$ follows the binomial distribution with parameters $n$ and $(1+\varepsilon) / 2$; that is,

$$
\mathbb{P}(X=k)=\left(\frac{1+\varepsilon}{2}\right)^{n-k}\left(\frac{1-\varepsilon}{2}\right)^{k}\left(\begin{array}{l}
n \\
k
\end{array}\right) \text {. }
$$

It is well known that the expected value and the variance of $X$ are given by $n \frac{1-\varepsilon}{2}$ and $n \frac{1-\varepsilon}{2} \frac{1+\varepsilon}{2}$, respectively. Hence,

$$
\begin{aligned}
\mathbb{E}\left(1-2 \frac{X}{n}\right)^{2} & =1-\frac{2}{n} \mathbb{E}(X)+\frac{1}{n^{2}} \mathbb{E}\left(X^{2}\right) \\
& =1-4 \frac{1-\varepsilon}{2}+\frac{4}{n^{2}}\left[\left(n \frac{1-\varepsilon}{2}\right)^{2}+n \frac{1-\varepsilon}{2} \frac{1+\varepsilon}{2}\right] \\
& >\left(1-2 \frac{1-\varepsilon}{2}\right)^{2}=\varepsilon^{2} .
\end{aligned}
$$

Thus, $\|U V\|_{c b} \geq N^{1 / 2} \varepsilon$.

Meanwhile, $\|V\| \leq N^{1 / 2} n^{\varepsilon-1 / 2}$. Set $\varepsilon \sim 1 / \log n$ (meaning, equality up to a universal constant), we obtain $\|U V\|_{c b}>N^{1 / 2} / \log n,\|V\|<N^{1 / 2} / n^{1 / 2}$.

The main result of this work follows from the proposition below. 
Proposition 4.2. Suppose $n$ is a positive power of 2. Then there exist $m \leq c n^{8}, s \leq n^{2}$ ( $c$ is an absolute constant), an operator $T: O H_{S} \rightarrow \ell_{1}^{m}\left(\ell_{\infty}^{n}\right)$, and a complete contraction $S: \ell_{1}^{m}\left(\ell_{\infty}^{n}\right) \rightarrow M_{n}$ so that

$$
\frac{\|S T\|_{c b}}{\|T\|}=\Omega\left(\frac{\sqrt{n}}{\log n}\right) .
$$

Moreover, one can select an orthonormal basis $\left(\theta_{i}\right)_{i=1}^{s}$ in $O H_{s}$ so that, for $1 \leq i \leq s, T \theta_{i}$ and $S T \theta_{i}$ have real coefficients relative to the canonical bases of the spaces $\ell_{1}^{m}\left(\ell_{\infty}^{n}\right)$ and $M_{n}$, respectively.

Now set

$$
M:=\frac{1}{\|T\|^{2}} \sum_{i=1}^{s} T \theta_{i} \otimes T \theta_{i} \in \ell_{1}^{m}\left(\ell_{\infty}^{n}\right) \otimes \ell_{1}^{m}\left(\ell_{\infty}^{n}\right) .
$$

According to Proposition 2.3, Corollary 2.4, (2.4), and (2.5), we have

$$
\|M\|_{\epsilon}=1 \quad \text { and } \quad\|M\|_{\min } \geq\|(S \otimes S) M\|_{M_{n} \otimes_{\min } M_{n}}=\Omega\left(\frac{n}{\log ^{2} n}\right) .
$$

Our main Theorem 1.1 follows now from Proposition 2.2.

Again, $T, S$, and $M$ depend on $n$, although this is not reflected in our notation (we wanted to avoid additional upper and lower indices).

Proof of Proposition 4.2. We need to "reduce the dimension" from $N$ to $m$. Let $H$ be the orthogonal complement of ker $U V$ in $O H_{N n}$, where $U$ and $V$ are the maps provided in Proposition 4.1. Note that $s:=\operatorname{dim} H \leq \operatorname{rank} U V \leq n^{2}$. Moreover, as $U V$ has real coefficients (with respect to the canonical bases), $H$ is the range of $(U V)^{t}$ (the transpose of $U V$ ). Consequently, $H$ is spanned by real linear combinations of the canonical basis of $O_{N n}$. A Gram-Schmidt procedure yields an orthonormal basis in $H$ whose elements have real coefficients relative to the canonical basis of $O H_{N n}$. Therefore, there exists an isometry $R: O H_{s} \rightarrow H$ with real coefficients (relative to the canonical basis $\left(\theta_{i}\right)_{i=1}^{s}$ of $O H_{s}$ and the canonical basis $\left(\delta_{x}\right)$ of $\left.O H_{N n}\right)$. Let $\tilde{V}=V R$. Then $\|\tilde{V}\| \leq\|V\|=O(\sqrt{N} / \sqrt{n})$, and due to the homogeneity of $O H_{N n}(2.6)\|U \tilde{V}\|_{c b}=\|U V\|_{c b}=$ $\Omega(\sqrt{N} / \log n)$. Let $E=\operatorname{Im} \tilde{V}$, which is an $n^{2}$-dimensional subspace of $\ell_{1}^{N}\left(\ell_{\infty}^{n}\right)$.

We will consider the space $S_{1}^{n}[E]$, which is known to be a subspace of $S_{1}^{n}\left[\ell_{1}^{N}\left(\ell_{\infty}^{n}\right)\right]$. Moreover, this last space is completely isometric to $\ell_{1}^{N}\left(S_{1}^{n}\left[\ell_{\infty}^{n}\right]\right)$ via the natural identification. Hence, if we denote $\tilde{E}=S_{1}^{n}[E]$ and $X=S_{1}^{n}\left[\ell_{\infty}^{n}\right]$, we have that the $n^{4}$ dimensional space $\tilde{E}$ is a subspace of $\ell_{1}^{N}(X)$. We can then apply Proposition 3.1 to deduce that the map $J$ introduced in Remark 3.1 verifies that $J \otimes i d_{X}: \ell_{1}^{N}(X) \rightarrow \ell_{1}^{m}(X)$ is a $\frac{1}{2}$-embedding when it is restricted to the subspace $\tilde{E}$ and $m \simeq n^{8}$. That is, the map $\left.\left(J \otimes \ell_{\infty}^{n}\right)\right|_{E} \otimes i d_{S_{1}^{n}}: S_{1}^{n}[E] \rightarrow \ell_{1}^{N}\left(S_{1}^{n}\left[\ell_{\infty}^{n}\right]\right)$ defines a $\frac{1}{2}$-isomorphism. The following diagram gives us the picture

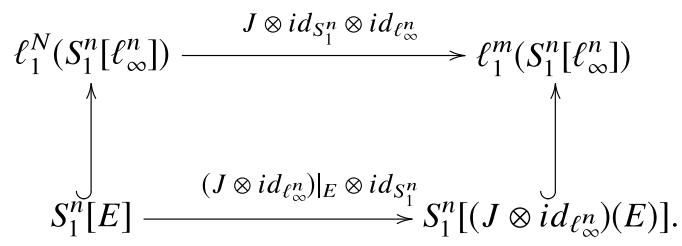

Let us define the linear map $T: O H^{n^{2}} \rightarrow \ell_{1}^{m}\left(\ell_{\infty}^{n}\right)$ given by $T=\left(J \otimes i d_{\ell_{\infty}^{n}}\right) \circ \tilde{V}$. Since $E=\operatorname{Im} \tilde{V}$ and $\left.\left(J \otimes i d_{\ell_{\infty}^{n}}\right)\right|_{E}$ must be an $\frac{1}{2}$-embedding, we immediately obtain that $\|T\| \lesssim\|\tilde{V}\|=O(\sqrt{N} / \sqrt{n})$. On the other hand, if we denote $F=\left(J \otimes i d_{\ell_{\infty}^{n}}\right)(E)$ we can define the map $S=U \circ\left(J^{-1} \otimes i d_{\ell_{\infty}^{n}}\right)$ : $F \rightarrow M_{n}$. Now, since $U$ is completely contractive and the map $\left.i d_{S_{1}^{n}} \otimes\left(J^{-1} \otimes i d_{\ell_{\infty}^{n}}\right)\right): S_{1}^{n}[F] \rightarrow$ $S_{1}^{n}\left[\ell_{1}^{N}\left(\ell_{\infty}^{n}\right)\right]$ is a $\frac{1}{2}$-embedding, we deduce that $\|S\|_{c b}=O(1)$. Indeed, to conclude this we have used that for every linear map $S$ into $M_{n}$ we have $\|S\|_{c b}=\left\|i d_{S_{1}^{n}} \otimes S\right\|$ (see Ref. 20, Theorem 1.5+Lemma 1.7). Moreover, we can extend $S$ to an operator from $\ell_{1}^{m}\left(\ell_{\infty}^{n}\right)$ to $M_{n}$ (also denoted by $S$ ) without increasing its cb norm (see Ref. 21, Corollary 1.7). 
Finally, note that

$$
\|S T\|_{c b}=\left\|U \circ\left(J^{-1} \otimes i d_{\ell_{\infty}^{n}}\right) \circ\left(J \otimes i d_{\ell_{\infty}^{n}}\right) \circ \tilde{V}\right\|_{c b}=\|U \tilde{V}\|_{c b}=\|U V\|_{c b}=\Omega\left(\frac{\sqrt{N}}{\log n}\right) .
$$

As $J$ has real (in fact, positive) coefficients, we are done.

\section{A. Some comments on our construction}

Let us finish this section with some final comments about our results. First, we notice that our procedure starts with the Khot-Vishnoi game $G_{K V}$, which has a particularly nice structure in $\ell_{1}^{N}\left(\ell_{\infty}^{n}\right) \otimes \ell_{1}^{N}\left(\ell_{\infty}^{n}\right)$ (it has for instance positive coefficients and it is completely explicit), and outputs another element $M \in \ell_{1}^{m}\left(\ell_{\infty}^{n}\right) \otimes \ell_{1}^{m}\left(\ell_{\infty}^{n}\right)$ with a more obscure description. There are several reasons for this. First of all, in order to apply the empirical method in the form of Proposition 3.1, we need to start with a low rank element, and the Khot-Vishnoi game has a very large rank. In order to overcome this obstacle, we must cut the Khot-Vishnoi game, which is done by considering the element $\tilde{V}=V R$ in the proof of Proposition 4.2, where $R$ is an isometry from $O H_{s}$ in $H$ with rank lower than or equal to $n^{2}$. Unfortunately, composing with the map $R$ involves a lack of control on the structure of the new object, since we do not have a complete description of $H$, the orthogonal complement of ker $U V$ in $O H_{N n}$. An important point is that we cannot assure that the new element $M$ (defined via $\tilde{V}=V R$ ) has positive coefficients when seen as an element in $\ell_{1}^{m}\left(\ell_{\infty}^{n}\right) \otimes \ell_{1}^{m}\left(\ell_{\infty}^{n}\right)$ (even though our starting point $G_{K V}$ did). This limitation is the main reason to consider the bias of the game (which allows us to work with nonpositive elements via the correspondence with Bell inequalities explained in the introduction). We think that proving a reduction atoms method which preserves positivity is a very interesting problem.

Another disadvantage of our result is that it introduces some randomness. Indeed, while the Khot-Vishnoi game is a completely explicit element in $\ell_{1}^{N}\left(\ell_{\infty}^{n}\right) \otimes \ell_{1}^{N}\left(\ell_{\infty}^{n}\right)$, our final element in $\ell_{1}^{m}\left(\ell_{\infty}^{n}\right) \otimes \ell_{1}^{m}\left(\ell_{\infty}^{n}\right)$ is not explicit since the map $J$ explained in Remark 3.1 has a probabilistic nature.

In order to obtain Theorem 1.1, we use Proposition 2.1, which does not rely on a specific quantum probability distribution to find a lower estimate for $\omega^{*}(M)$. However, inspecting the proof of Proposition 4.2, one can see that our lower estimate is witnessed by the $n$-dimensional maximally entangled state.

The procedure used in this paper is very general and it can be applied in many different contexts. There are two key points in our proof. First of all, the Khot-Vishnoi game is an $\mathrm{OH}$-game, in the sense that it can be seen as an element of the form $V V^{*}$, where $V: O H_{N n} \rightarrow \ell_{1}^{N}\left(\ell_{\infty}^{n}\right)$. The second crucial element in our proof is that although the game $G_{K V}$ has a very large rank, the dimension used in the corresponding quantum strategy to lower bound the value $\omega^{*}\left(G_{K V}\right)$ is of order $n$. The previous two ingredients allow to "cut the Khot-Vishnoi game" so that we obtain a new element with lower rank and essentially the same classical and quantum values. Although factoring through a Hilbert space $O H_{N}$ can be understood as a very restrictive property, most of the games used to obtain large Bell violations have this characteristic (as those in Refs. 10 and 11). Hence, this property seems to be very natural when studying extreme objects and it is very plausible that our method is of independent interest in some other contexts such that the study of integrality gaps between the classical and quantum value of a game and certain SDP-relaxations.

Finally, we note that exactly the same approach followed in this work can be applied to the recent paper ${ }^{18}$ to prove that Bell violations of order $\sqrt{n} / \log ^{2} n$ can be obtained by only using binary questions for one party and with the additional property that only a polynomial number of questions are needed.

\section{ACKNOWLEDGMENTS}

The first author was partially supported by the NSF DMS-1201886. The second author was partially supported by Simons Foundation travel Award No. 210060. The third author was partially supported by MINECO (Grant No. MTM2014-54240-P), QUITEMAD+-CM, (S2013/ICE-2801) funded by Comunidad de Madrid, the European CHIST-ERA project CQC (funded partially by 
MINECO Grant No. PRI-PIMCHI-2011-1071), and "Ramón y Cajal" program. The first and third authors are partially supported by ICMAT Severo Ochoa Grant No. SEV-2011-0087 (Spain).

${ }^{1}$ Aspect, A., Grangier, P., and Roger, G., "Experimental tests of realistic local theories via Bell's theorem,” Phys. Rev. Lett. 47(7), 460-463 (1981).

2 Bell, J. S., “On the Einstein-Poldolsky-Rosen paradox,” Physics 1(3), 195 (1964).

${ }^{3}$ Bourgain, J., Lindenstrauss, J., and Milman, V., "Approximation of zonoids by zonotopes," Acta Math. 162, $73-141$ (1989).

${ }^{4}$ Buhrman, H., Regev, O., Scarpa, G., and de Wolf, R., "Near-optimal and explicit Bell inequality violations,” Theory Comput. 8, 623-645 (2012).

${ }^{5}$ Clauser, J. F., Horne, M. A., Shimony, A., and Holt, R. A., "Proposed experiment to test local hidden-variable theories," Phys. Rev. Lett. 23, 880 (1969).

${ }^{6}$ Cleve, R., Høyer, P., Toner, B., and Watrous, J., "Consequences and limits of nonlocal strategies," in IEEE 19th Annual Conference on Computational Complexity (IEEE, 2004), pp. 236-249.

${ }^{7}$ Effros, E. and Ruan, Z.-J., Operator Spaces, London Mathematical Society Monographs New Series Vol. 23 (Oxford University Press, Oxford, 2000).

${ }^{8}$ Einstein, A., Podolsky, B., and Rosen, N., "Can quantum-mechanical description of physical reality be considered complete?," Phys. Rev. 47, 777 (1935).

${ }^{9}$ Hensen, B., Bernien, H., Dréau, AE., Reiserer, A. et al., "Experimental loophole-free violation of a Bell inequality using entangled electron spins separated by $1.3 \mathrm{~km}$," Nature 526(7575), 682-686 (2015).

10 Junge, M. and Palazuelos, C., "Large violation of Bell inequalities with low entanglement," Commun. Math. Phys. 306(3), 695-746 (2011).

${ }^{11}$ Junge, M., Palazuelos, C., Pérez-García, D., Villanueva, I., and Wolf, M., "Unbounded violations of bipartite Bell inequalities via operator space theory," Commun. Math. Phys. 300, 715-739 (2010).

12 Junge, M., Palazuelos, C., Pérez-García, D., Villanueva, I., and Wolf, M., "Operator space theory: A natural framework for Bell inequalities," Phys. Rev. Lett. 104, 170405 (2010).

${ }^{13}$ Khot, S. and Vishnoi, N., "The unique games conjecture, integrality gap for cut problems and embeddability of negative type metrics into $\ell_{1}$," in Proceedings of 46th IEEE FOCS (IEEE, 2005), pp. 53-62.

${ }^{14}$ Loubenets, E. R., "Local quasi hidden variable modelling and violations of Bell-type inequalities by a multipartite quantum state," J. Math. Phys. 53, 022201 (2012).

15 Palazuelos, C., "Superactivation of quantum nonlocality," Phys. Rev. Lett. 109, 190401 (2012).

${ }^{16}$ Palazuelos, C., "On the largest Bell violation attainable by a quantum state," J. Funct. Anal. 267, 1959-1985 (2014).

${ }^{17}$ Palazuelos, C. and Vidick, T., "Survey on nonlocal games and operator space theory," J. Math. Phys. 57, 015220 (2016).

${ }^{18}$ Palazuelos, C. and Yin, Z., "Large bipartite Bell violations with dichotomic measurements," Phys. Rev. A 92, 052313 (2015).

${ }^{19}$ Pisier, G., "The operator Hilbert space $\mathrm{OH}$, complex interpolation and tensor norms," Mem. Am. Math. Soc. 122, 585 (1996).

${ }^{20}$ Pisier, G., "Non-commutative vector valued $L_{p}$-spaces and completely $p$-summing maps," Astérisque No. $247,1998$.

${ }^{21}$ Pisier, G., Introduction to Operator Space Theory, London Mathematical Society Lecture Note Series Vol. 294 (Cambridge University Press, Cambridge, 2003).

${ }^{22}$ Schechtman, G., "More on embeddings subspaces of $L_{p}$ in $\ell_{r}^{n}$," Compositio Math. 61, 159-170 (1987).

23 de Wolf, R., "A brief introduction to fourier analysis on the boolean cube," Theory of Computing Library Graduate Surveys TCGS 1, 1-20 (2008).

${ }^{24}$ The situation where the players maximize the value of the game is also very interesting, but the optimization of the bias is more suitable in this work.

${ }^{25}$ In Ref. 4 the authors study the ratio of the quantum over the classical value of the game rather than the bias. 